\title{
Impact of Initial Prognostic Factors and Intensity of Salvage Therapy on the Outcome of Progressive I Refractory High-Risk Neuroblastoma
}

\author{
Ahmed Elhemaly ${ }^{1, *}$, Ahmed Fathalla ${ }^{2}$, Mahmed Elhusseny ${ }^{3}$, Mohamed Fawzy ${ }^{1}$ \\ ${ }^{1}$ Pediatric Oncology, National Cancer Institute (NCI)-Cairo University and Children Cancer Hospital of Egypt (CCHE), Cairo, Egypt \\ ${ }^{2}$ Surgical Oncology, National Cancer Institute, Cairo, Egypt \\ ${ }^{3}$ Pediatric Oncology, Children cancer Hospital of Egypt, Cairo, Egypt
}

Email address:

ahmed.ibrahiem@57357.org (A. Elhemaly), Drasf1975@gmail.com (A. Fathalla), muhammed.elhusseiny@57357.org (M. Elhusseny), mfawzyonc@cu.edu.eg (M. Fawzy)

${ }^{*}$ Corresponding author

To cite this article:

Ahmed Elhemaly, Ahmed Fathalla, Mahmed Elhusseny, Mohamed Fawzy. Impact of Initial Prognostic Factors and Intensity of Salvage Therapy on the Outcome of Progressive / Refractory High-Risk Neuroblastoma. Cancer Research Journal. Vol. 9, No. 2, 2021 , pp. 85-91. doi: $10.11648 /$ j.crj.20210902.11

Received: March 13, 2021; Accepted: March 27, 2021; Published: April 7, 2021

\begin{abstract}
Background: High-risk Neuroblastoma (N. B) patients have a poor outcome with 5-year survival rates of 50\%. Patients with stage 4 disease or MYC-N amplification showed post-progression 5y O. S. of 7\% to $8 \%$. Other studies proved the same dismal outcome in high-risk relapsed patients. This study aimed to detect the O. S. and EFS of N. B patients postprogression. Secondary to explore, if initial prognostic factors, high-intensity salvage therapy and other treatment modalities could improve the outcome of progressive /refractory disease. Methods: Seventy patients of high-risk Neuroblastoma needed salvage therapy, either due to refractory/progressive disease or irresectability of the primary tumor. Initial prognostic factors and different treatment strategies were collected and correlated with the outcome. Results: Fifty-seven (57/70) patients died from progressive disease with a median survival of 20.6 months with three y EFS and O. S. of $9.5 \%$ and $35.7 \%$, respectively. Objective response (CR/VGPR/PR) post-induction, consolidation by HSCT, radiotherapy, and maintenance therapy; affected survival significantly post salvage therapy. Multivariate analysis revealed that the only independent factor that significantly affected O. S was maintenance therapy. The independent factors that affected the EFS negatively were the presence of liver metastases, poor response post-induction, and not administering radiotherapy. Conclusion: Response to induction had a significant impact on the outcome post salvage. Salvage therapy did not improve the outcome for those with inadequate induction response. Initial front-line targeted therapy like antiGD2 is needed to improve the outcome, especially for chemoresistant ones.
\end{abstract}

Keywords: Salvage, Neuroblastoma, High Risk-Low Middle, Income Countries

\section{Introduction}

Neuroblastoma (N. B.) is an embryonal malignancy arising from neuroblasts of the sympathetic nervous system. Patients with metastatic disease or high-risk biologic features have a poor outcome, with $50 \%$ survival at five years [1].

Age, stage, histopathology, and biological features (MYC$\mathrm{N}$ gene status, DNA ploidy) are the prognostic factors affecting the outcome [2]. Many studies were carried out to improve the outcome of this aggressive disease by using myeloablative conditioning, followed by stem cell rescue. Mega therapy showed improvement in event-free survival (EFS) compared to conventional treatment [3]. Treatment of high-risk Neuroblastoma consists of induction chemotherapy, local control by surgery, consolidation by autologous transplant, radiotherapy followed by maintenance with both immunotherapy and a differentiating agent (Isotretinoin) [3]. Cis retinoic acid and immunotherapy are used to eradicate minimal residual disease [4]. N. B is an aggressive disease with a significant portion showed refractory/progressive 
disease or inadequate response. The most common salvage regimen used is the ICE regimen (Ifosamamide, Carboplatin, and Etoposide) [5]. Patients with stage 4 disease or MYCN amplification showed post-progression $5 \mathrm{y} \mathrm{O}$. S. of $7 \%$ to $8 \%$. Other studies proved the same dismal outcome in high-risk relapsed patients $[6,7]$. This study aimed to detect O. S. and EFS of N. B. patients post-progression. The Secondary aim was to explore if the initial prognostic factors, high-intensity salvage therapy, and other treatment modalities could improve the outcome of progressive /refractory disease.

\section{Methods}

The present study included seventy (70) patients diagnosed with $\mathrm{N}$. B, with a progressive or refractory disease in the period between January 2007 and December 2015 and were followed till December 2017. All patients were younger than 18 years of age and diagnosed with high-risk N. B. classified according to COG ANBLOOB1 risk stratification. SFOP NB 97 protocol was given as induction for fear of toxicities from the more aggressive COG A3973 protocol. Progression was defined as the appearance of new lesion or $25 \%$ increase in any existing lesion after achieving CR/ VGPR [8]. Refractory patients included those who had not achieved at least a P. R. to induction therapy (minimum of four cycles) and did not show progression [9]. Initial prognostic factors like age, initial tumor site, pathology, MYCN status, stage, different metastatic sites, extent of resection, response to induction, tumor viability, consolidation, and maintenance therapy post salvage were analyzed and correlated with outcome post salvage.

\subsection{Treatment}

All patients received induction, 4-8 cycles of Vp16/CARBO (Etoposide, Carboplatin) alternating with CADO (Cyclophosphamide, Adriamycin, Vincristine) according to SFOP NB90 protocol [10]. Patients were evaluated after four cycles and at the end of treatment. International Neuroblastoma Response Criteria (INRC) was used to stratify response. If feasible, surgery was done after four cycles.

\subsection{Definitions}

Tumor resectability was classified as complete resection (95-100\%), gross total resection (90-95\%), incomplete resection (50-90\%), and biopsy $(<50 \%)$ [11]. Patients with refractory /progressive disease or significant local residual were subjected to salvage chemotherapy ICE regimen (Ifosfamide, Carboplatin. Etoposide) for 6-8 cycles. Patients who achieved (C. R., VGPR, or P. R.) post salvage, did stem cell collection followed by myeloablative conditioning BU/MEL (Busulfan, Melphalan), then auto BMT. The primary tumor site and residual metastatic sites postinduction (up to 5 sites) received fractionated irradiation (2100 cGy if no local residual or $3600 \mathrm{cGy}$ if gross residual) around $\mathrm{D}+45$ post auto BMT or post salvage therapy (if they were not transplanted) followed by 12 cycles of Cis-Retinoic acid as maintenance therapy.

\section{Statistical Analysis}

Statistical analysis was done using SPSSC Statistics version 22. Survival analysis was done using the KaplanMeier method. The log-rank test was used to compare two survival curves. A p-value $<0.05$ was considered significant. Event-free survival post-progression (EFS): was defined as the time between diagnosis of first progression/relapse to the date of any event (progression or relapse or death). O. S. post-progression was defined as the time between diagnosis of first progression/relapse and death or date of the last follow-up. Multivariate analysis was done using the Coxproportional Hazard regression model for the significant factors affecting the survival on univariate analysis. Hazard ratio (H. R.), with its $95 \%$ confidence interval $(\mathrm{CI})$, was used for risk estimation.

\section{Results}

The study included 70 patients with progressive/refractory high-risk N. B. Patients' age ranged from 4 to 120 months (median: 36 months $\pm 22.8 \mathrm{SD}$ ) with slight male predominance with $\mathrm{M} / \mathrm{F}$ ratio $1.29: 1$, All clinic pathological data were summarized in table 1. Salvage therapy (ICE) was given due to local inoperability in $11(15.7 \%)$ patients, refractory disease at the end of induction in $26(37.1 \%)$ patients, and due to progressive disease in 33 (47.1\%) patients. Figure 1.

Different prognostic factors like age, tumor site, stage, histopathology (favorable/unfavorable), MYC-N status, and initial serum ferritin did not show a significant impact on outcome post salvage therapy. (for details table 2). At relapse/progression, resection of primary tumor- regardless of the extent- was associated by significant positive impact on survival with $3 y$ EFS of $12.8+/-5.4$ and $8.1 \%+/-6.6 \%$ for the surgery and no surgery group, respectively (P-value $<0.01$ ); and $3 y$ OS of $36.7 \%+/-8 \%$ and $23.2 \%+/-8.3 \%$, respectively with borderline significance (p-value 0.07$)$. Thirty-eight (38/70) patients showed a post-induction objective response (C. R., VGPR, PR), while 32/70 showed (N. R., P. D.). The 3y postprogression EFS and OS for responders were 14.4\% $+/-6.3 \%$ and $47.6+/-8.5 \%$, respectively; while the $3 y$ EFS and OS for non responders were $3.1 \%+/-3.1 \%$ and $21.9 \%+/-7.3 \%$, respectively (P-value for EFS was $<0.001$ and for OS was $<0.01$. Tumor histological chemotherapy effect (differentiation $>10 \%$ ) was associated with a significant positive impact on both EFS (P-value $<0.005$ ) and O. S. (Pvalue 0.05 ). The presence of metastatic disease (lymph node, bone marrow, bone, lung, distant lymph node) was associated with lower post-progression EFS and O. S. but without statistical significance (Table 2). Presence of liver metastatic disease was associated with lower 3 y EFS and OS of $0 \%$ and $14.3 \%+/-13.2 \%$, respectively compared to $10.6 \%+/-4.2 \%$ and $38 \%+/-6.3 \%$ respectively for those without (P-value 
$<0.001$ for EFS and 0.07 for OS). Sixteen (16/70) (22.8\%) underwent HSCT after achieving CR/VGPR/PR. Five (5/16) patients continued in CR/VGPR post-BMT, while 11 patients showed progressive disease. Fifty-four patients did not achieve objective response post salvage and did not do the transplant; 53/54 patients showed progressive disease. The median survival of the transplanted group was 17.8 months versus 8.9 months for the non transplanted group. The 3 y EFS and OS for the non transplanted group were $4.4 \%+/-3 \%$ and $24.9 \%$ $+/-6 \%$, respectively compared to $26.3 \%+/-12.1 \%$ and $62.9 \%$
+/- 13.8\% for transplanted group, respectively (P-value 0.002 for EFS and 0.003 for O. S). Local RTH was given in 16/70 $(22.8 \%)$ with $3 y$ EFS and OS of $36.5 \%+/-13.6$ and $85.1 \%+/-$ $9.7 \%$, respectively for RTH group compared to $1.9+/-1.8 \%$ and $21.6 \%+/-5.7 \%$, respectively for those who did not receive RTH (P-value $<0.001$ for both EFS and OS). Nineteen (19/70) $(27.1 \%)$ patients received 12 cycles of Cis-retinoic acid as maintenance therapy with a significant positive impact on survival (P-value for EFS and O. S. $<0.001$ ).

Table 1. Clinico pathological data of the studied patients.

\begin{tabular}{|c|c|c|}
\hline Variable & Number Total $\mathrm{n}=\mathbf{7 0}$ & Percentage (\%) \\
\hline \multicolumn{3}{|l|}{ Primary site } \\
\hline Adrenal & 57 & $81.4 \%$ \\
\hline Abdominal (non-adrenal) & 11 & $15.7 \%$ \\
\hline Mediastinum & 1 & $1.4 \%$ \\
\hline Neck & 1 & $1.4 \%$ \\
\hline \multicolumn{3}{|l|}{ Stage (INSS) } \\
\hline Stage $2 / 3$ & 11 & $15.7 \%$ \\
\hline Stage 4 & 59 & $84.3 \%$ \\
\hline \multicolumn{3}{|l|}{ Pathology } \\
\hline NB & 68 & $97.14 \%$ \\
\hline GNB & 2 & $2.8 \%$ \\
\hline \multicolumn{3}{|l|}{ INPC } \\
\hline Favourable & 10 & $14.28 \%$ \\
\hline Unfavourable & 60 & $85.7 \%$ \\
\hline \multicolumn{3}{|l|}{ MYCN } \\
\hline Amplified & 18 & $25.7 \%$ \\
\hline Non-amplified & 23 & $32.8 \%$ \\
\hline Not-applicable & 29 & $41.4 \%$ \\
\hline \multicolumn{3}{|l|}{ Ferritin } \\
\hline Normal & 43 & $61.4 \%$ \\
\hline Elevated & 27 & $38.5 \%$ \\
\hline \multicolumn{3}{|l|}{ Surgery } \\
\hline Yes & 39 & $55.7 \%$ \\
\hline No & 31 & $44.2 \%$ \\
\hline \multicolumn{3}{|l|}{ Chemotherapy effect } \\
\hline$<10 \%$ & 13 & $41.9 \%$ \\
\hline$>10 \%$ & 18 & $58 \%$ \\
\hline \multicolumn{3}{|l|}{ Metastatic sites } \\
\hline \multicolumn{3}{|l|}{ BM } \\
\hline No & 17 & $24.28 \%$ \\
\hline Yes & 53 & $75.7 \%$ \\
\hline \multicolumn{3}{|l|}{ Bone } \\
\hline No & 45 & $64.2 \%$ \\
\hline Yes & 25 & $35.7 \%$ \\
\hline \multicolumn{3}{|l|}{ LNs } \\
\hline No & 59 & $84.28 \%$ \\
\hline Yes & 11 & $15.71 \%$ \\
\hline \multicolumn{3}{|l|}{ CNS } \\
\hline No & 60 & $85.7 \%$ \\
\hline Yes & 10 & $14.28 \%$ \\
\hline \multicolumn{3}{|l|}{ Liver } \\
\hline No & 63 & $90 \%$ \\
\hline Yes & 7 & $10 \%$ \\
\hline
\end{tabular}

INSS: international neuroblastoma staging system, LNS: lymph nodes, CNS: central nervous system, INPC: international neuroblastoma pathology classification 
Ahmed Elhemaly et al: Impact of Initial Prognostic Factors and Intensity of Salvage Therapy on the Outcome of Progressive / Refractory High-Risk Neuroblastoma

Table 2. Correlation between different prognostic factors and O. S and EFS.

\begin{tabular}{|c|c|c|c|c|}
\hline & OS at 36 months & $P$ - value & EFS at 36 months & p-value \\
\hline \multicolumn{5}{|l|}{ Age } \\
\hline$<12$ months & $20+/-17.9 \%$ & \multirow{2}{*}{ NA } & $0 \%$ & \multirow{2}{*}{ NA } \\
\hline$>18$ & $39.3 \%+/-6.5 \%$ & & $10.9 \%+/-4.3$ & \\
\hline \multicolumn{5}{|l|}{ Ferritin } \\
\hline Normal & $21.9+/-7.3 \%$ & \multirow[t]{2}{*}{0.27} & $11.2+/-5.4 \%$ & \multirow[t]{2}{*}{0.23} \\
\hline High & $29.6+/-8.8 \%$ & & $7.4+/-5 \%$ & \\
\hline \multicolumn{5}{|l|}{ Site: } \\
\hline Adrenal & $43.6+/-6.6 \%$ & \multirow{3}{*}{ NA } & $6.8+/-3.6 \%$ & \multirow{3}{*}{ NA } \\
\hline Abdominal (non-adrenal) & $45.5 \%+/-15 \%$ & & $24.2+/-13.8 \%$ & \\
\hline Mediastinum & $0 \%$ & & $57.1 \%$ & \\
\hline \multicolumn{5}{|l|}{ Stage: } \\
\hline II\&III & $36.4+/-14.5 \%$ & 0.40 & $27.3+/-13.4 \%$ & 0.42 \\
\hline \multicolumn{5}{|l|}{ Histology: } \\
\hline FH & $40+/-15.5 \%$ & \multirow[t]{2}{*}{0.57} & $10+/-9.5 \%$ & \multirow[t]{2}{*}{0.64} \\
\hline UFH & $35.1+/-6.4 \%$ & & $9.7+/-4.2 \%$ & \\
\hline \multicolumn{5}{|l|}{ MYCN: } \\
\hline Amplified & $14.8+/-8.9 \%$ & \multirow[t]{2}{*}{0.55} & $5.6+/-5.4 \%$ & \multirow[t]{2}{*}{0.305} \\
\hline Not amplified & $41.1+/-10.8 \%$ & & $14.5+/-8.2 \%$ & \\
\hline \multicolumn{5}{|l|}{ Metastatic sites: } \\
\hline $\mathrm{BM}$ & & & & \\
\hline No (17) & $30.9+/-12.6 \%$ & 0.78 & $17.6+/-9.2 \%$ & 0.61 \\
\hline Yes (53) & $36.9+/-6.7 \%$ & & $8.4+/-3.9 \%$ & \\
\hline Bone & & & & \\
\hline No (45) & $30.6+/-7.3 \%$ & 0.66 & $9.3+/-4.7 \%$ & 0.65 \\
\hline Yes (25) & $44.4+/-9.9 \%$ & & $12+/-6.5 \%$ & \\
\hline Yes (10) & $50+/-15.8 \% ? /$ & & $10+/-9.5 \%$ & \\
\hline Liver & & & & \\
\hline No (63) & $38+/-6.3 \%$ & 0.07 & $10.6+/-4.2 \%$ & 0.001 \\
\hline Yes (7) & $14.3+/-13.2 \%$ & & $0 \%$ & \\
\hline Lymph node & & & & \\
\hline No (59) & $38.1+/-6.4 \%$ & 0.28 & $8.5+/-3.9 \%$ & 0.84 \\
\hline Yes (11) & $18.2+/-14.8 \%$ & & $18.2+/-11.6 \%$ & \\
\hline Status post induction & & & & \\
\hline CR/VGPR/PR (38) & $47.6+/-8.5 \%$ & 0.018 & $14.4+/-6.3 \%$ & $<0.001$ \\
\hline NR/PD (32) & $21.9+/-7.3 \%$ & & $3.1+/-3.1$ & \\
\hline Surgery & & & & \\
\hline Yes (39) & $36.7+/-8 \%$ & 0.07 & $12.8+/-5.4 \%$ & 0.01 \\
\hline No (31) & $23.2+/-8.3 \%$ & & $8.1+/-6.6$ & \\
\hline Chemotherapy effect & & & & \\
\hline$<10 \%$ & $23.1+/-11.7 \%$ & 0.05 & $0 \%$ & 0.005 \\
\hline$>10 \%$ & $58.7 \%+/-13 \%$ & & $20+/-10.3 \%$ & \\
\hline HSCT & & & & \\
\hline No (54) & $24.9+/-6 \%$ & 0.003 & $4.4+/-3 \%$ & 0.002 \\
\hline Yes (16) & $62.9+13.8 \%$ & & $26.3+/-12.1 \%$ & \\
\hline RTH & & & & \\
\hline No (54) & $21.6+/-5.7 \%$ & $<0.001$ & $1.9+/-1.8 \%$ & $<0.001$ \\
\hline Yes (16) & $85.1+/-9.7 \%$ & & $36.5+/-13.6 \%$ & \\
\hline Cis-retinoic acid & & & & \\
\hline No (51) & $16.9+/-5.3 \%$ & $<0.001$ & $2+/-1.9 \%$ & $<0.001$ \\
\hline Yes (19) & $79.9+/-10.6 \%$ & & $30.7+/-11.8 \%$ & \\
\hline
\end{tabular}

BM: bone marrow, CNS: central nervous system, FH: favorable, UFH: unfavorable, HSCT: hematopoietic stem cell transplant, RTH: radiotherapy 


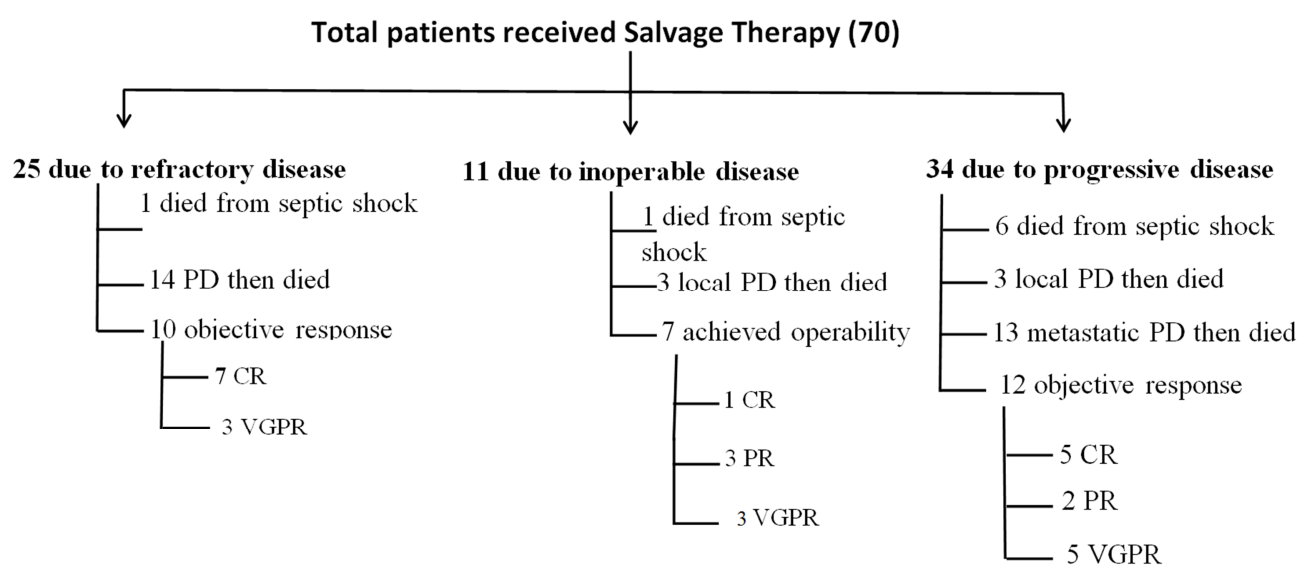

Figure 1. Outcome of studied patients post salvage.

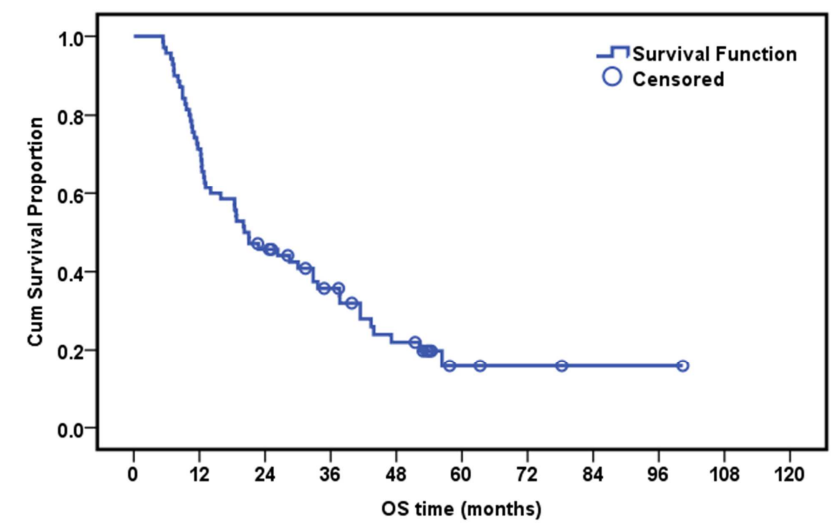

Figure 2. O. S of the whole studied group.

\subsection{Outcome of Salvage Therapy}

Eight patients died from septic shock. Sixty-two patients were evaluated; thirteen patients $(21 \%)$ achieved C. R. Twenty-nine patients (46.7\%) showed an objective response. Thirty-three $(53.3 \%)$ patients showed progressive disease (Figure 1). The included patients were followed for a median duration of 22 months (ranged $2.3-115.9$ months). The median O. S. and EFS of the whole group were 20.6 and 9.6 months, respectively. The cumulative O. S. and EFS $35.7 \%$ and $9.5 \%$ at 36 months, as shown in (Figures 2 and 3 ).

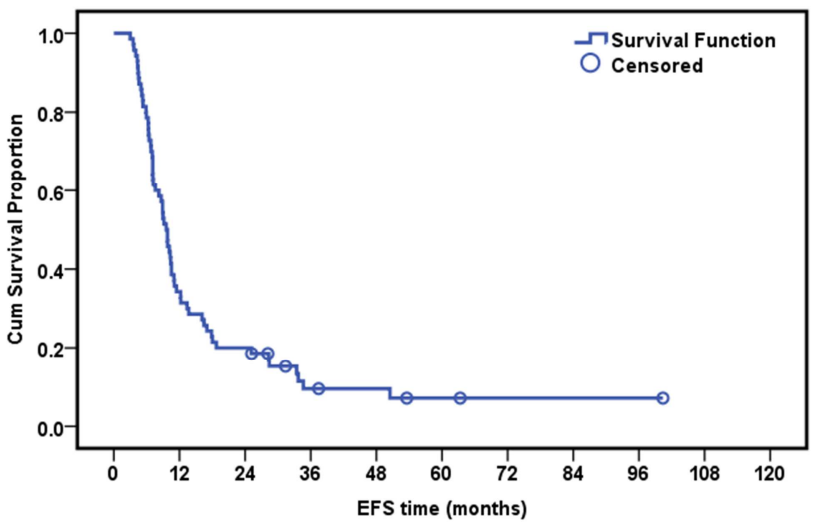

Figure 3. EFS of the whole studied group.

\subsection{Correlation Between Different Prognostic Factors with O. S. and EFS}

Response at the end of induction, consolidation by HSCT, radiotherapy, and Cis-Retinoic acid as maintenance had a significant impact on the outcome. Multivariate analysis revealed that the only independent factor that significantly affected the O. S. was the maintenance therapy (H. R.: 8.6, 95\% CI (3.4-21.9); $\mathrm{p}<0.001)$. The independent factors that significantly affected the EFS negatively were the presence of liver metastases (H. R.: 3.5, 95\% CI $(1.5-8.1) ; p=0.004)$, the inadequate response at the end of induction (N. R., P. D.) (H. R.: $3.1,95 \%$ CI (1.7-.5); $\mathrm{p}<0.001)$ and no radiotherapy (H. R.: 3.1, 95\% CI (1.4-6.9); $\mathrm{p}=0.006$ ) (Figures 4 and 5).

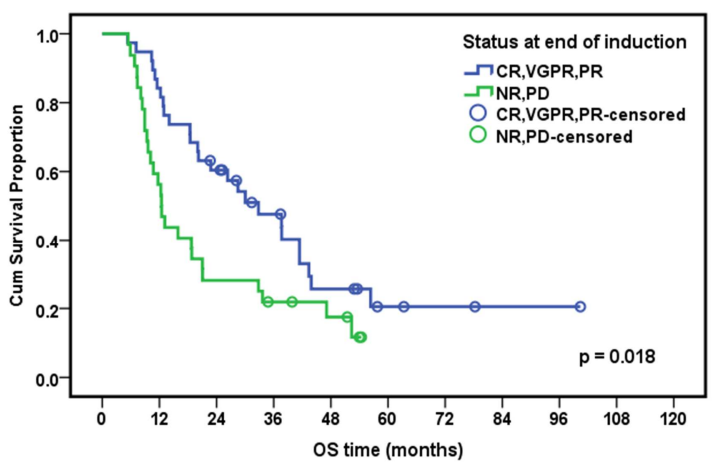

Figure 4. Correlation between post induction response and OS of the studied patients.

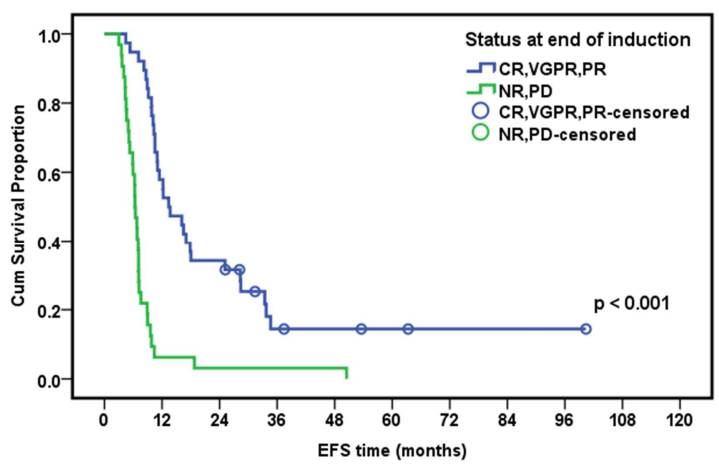

Figure 5. Correlation between post induction response and EFS of the studied patients. 


\section{Discussion}

In high-risk N. B., the outcome is more correlated to the biological characteristics than the type of chemotherapy or extent of resection [12]. The poorer prognosis was associated with the high serum ferritin, bone, and B. M. metastasis, as well as to persisting skeletal and B. M. disease post-induction therapy [13]. There is no much data in the literature about post-progression O. S. and PFS. In the present study, patients with amplified MYC-N gene showed lower 3y EFS and O. S. compared to non amplified ones, although it showed clinical significance, it lacked statistical significance due to small numbers. Jennifer concluded that the only disease characteristics associated with worse 5-year O. S. postprogression were MYC-N amplification and multifocal soft tissue disease [14]. In the present study, initial metastatic sites did not affect the survival except for metastatic liver disease. Basta reported that MYC-N amplification $(\mathrm{P}<0.0001)$ and liver metastases $(\mathrm{P}=0.02)$ were the only factors associated with worse post relapse O. S. in the univariate analysis [15]. In contrast, MYC-N status and presence of bone marrow disease were the only independent poor prognostic factors that affected O. S. (P value=0.007 for both).

Response to induction therapy was proven to influence the outcome of high-risk Neuroblastoma [16]. There is no much data in the literature about the impact of response to induction on relapsing/progressive disease outcomes. In the present study, it was evident that patients with good induction response (C. R., VGPR, PR) showed better survival even when they progressed and were salvaged compared to those with inadequate induction response. Patients who achieved objective response post-induction $(\geq \mathrm{P}$. R.) had significantly better three y post-progression EFS and O. S. compared to those with less than P. R. response ( $\mathrm{P}$ value $<0.001$ for EFS, $<0.018$ for $\mathrm{O}$. S.). Response to salvage therapy is dependent -in our point of view- on the tumor biology, which affects tumor response initially and at relapse/progression. Intensification of induction/salvage may not improve the outcome of high-risk N. B. with poor biological features as they have poor outcome whatever kind of therapy given.

ICE chemotherapy was given for all patients with a progressive or refractory disease with $38.4 \%$ of the refractory patients showed an objective response (C. R., VGPR) compared to $35.2 \%$ of the patients in the progressive group. Kushner et al. who used ICE as salvage, reported significant responses (C. R., VGPR, and P. R.) in 53\% of relapsing patients versus $15 \%$ in patients with refractory N. B. and just in $3 \%$ of patients with P. D [5].

An older study by Abdel Rahman reported better cumulative O. S. of $65 \%$ for progressive N. B. patients and $68 \%$ for refractory ones who received ICE regimen and less treatmentrelated mortality (3\%) with objective response $36.5 \%$ compared to our results (OS 35.7\% at three years) [17].

Consolidation by hematopoietic stem cell transplant improved outcome significantly post salvage therapy compared to non-transplanted group (P-value 0.002 for EFS and 0.003 for O. S.). A study carried by Simon et al.; demonstrated better median survival of 2.08 and 3-year O. S. of $43.5 \pm 10.9 \%$ for the transplanted group compared to those who did not undergo ASCT (median survival of 0.89 years, 3year survival rate of $9.6 \pm 2.8 \mathrm{P}<0.001)$ [18].

Additional studies suggested that haploidentical stem cell transplantation may improve the outcome of children with relapsed and refractory N. B [19]. In the current study, objective response post-induction, consolidation by HSCT, radiotherapy, and maintenance post salvage showed a significant positive impact on the outcome. The presence of metastatic liver disease affected EFS negatively. Simultaneously, multivariate analysis showed that maintenance therapy was the only independent factor affecting the O. S. The independent factors that affected the EFS were the presence of liver metastases, the inadequate response at the end of induction (N. R., P. D.) and not administrating radiotherapy. A similar Italian study by Alberto Garverneta et al. proved that age less than 18 months, extra-abdominal primary, lower stage, and absence of MYC$\mathrm{N}$ amplification had a significant positive impact on $\mathrm{O}$. S. and the multivariate analysis revealed that age, stage, and MYC-N status are the independent prognostic factors [8].

In our view, improving the outcome in high-risk N. B. is not by intensifying induction/salvage as the problem is the tumor biology, which predicts tumor response. Major Responders (C. R., VGPR, PR) to low-intensity induction have better response to salvage therapy than poor or non-responders even if they received high-intensity salvage therapy.

\section{Conclusions}

Our results emphasize that N. B. is a salvageable disease. ICE chemotherapy is a good salvage regimen. More concerns should be paid for supportive measures. Response to induction had a significant impact on the outcome post salvage. Salvage therapy did not improve the outcome for those with inadequate induction response. Consolidation by HSCT, radiotherapy, and maintenance therapy improved the outcome for responders to salvage therapy. Initial front-line targeted therapy like antiGD2 is needed to improve the outcome, especially for chemo-resistant ones who do not benefit much from therapy intensification.

\section{Declarations}

This study was not funded by any organization and was approved by our hospital' scientific and ethical committee; the authors declared that they did not have any conflict of interest.

\section{Acknowledgements}

This study was not funded by any organization and was approved by our hospital' scientific and ethical committee; the authors declared that they did not have any conflict of interest 


\section{References}

[1] Park JR, Bagatell R, London WB, et al (2013). Children Oncology Group's blueprint for research: Neuroblastoma. Pediatric blood cancer, 60, 985-3.

[2] Look AT, Hayes FA, Shuster JJ, et al (1991). Clinical relevance of tumor cell ploidy and $\mathrm{N}$-myc gene amplification in childhood neuroblastoma: a Pediatric Oncology Group study. J Clin Oncol, 9, 581-1.

[3] Parikh NS, Howard SC, Chantada G, et al (2015). SIOPPODC adapted risk stratification and treatment guidelines: Recommendations for Neuroblastoma in low-and-middleincome settings. Pediatric blood cancer, 62, 1305-6.

[4] Greengard E, Hill KC, Bagatell R, (2013). Treatment of highrisk Neuroblastoma in children: recent clinical trial results. Clinical. Invest, 3, 1071-1.

[5] Kushner H B, Modak S, Kramer K, et al (2013). High-dose salvage regimen and review of the literature. Cancer, 119, 665-1.

[6] Lau L, Tai D, Weitzman S, et al (2004). Factors influencing survival in children with recurrent Neuroblastoma. J Pediatr Hematol Oncol, 26, 227-2.

[7] Santana VM, Furman WL, McGregor LM, Catherine A Billups, (2008). Disease control intervals in high-risk Neuroblastoma. Cancer, 112, 2796-1.

[8] Garaventa A, Parodi S, De Bernardi B, et al (2009). The outcome of children with Neuroblastoma after progression or relapse. A retrospective study of the Italian neuroblastoma registry, Eur J Cancer 45, 2723-2.

[9] Zhou JM, Doral YM, Du Bois SG, et al (2015). Different outcomes for relapsed vs. refractory Neuroblastoma after therapy with 131I-metaiodobenzylguanidine (131I-MIBG). Eur J Cancer, 51, 2465-2.

[10] Bergeron C, Dubourg L, Chastagner P, et al (2005). Long-term renal and hearing toxicity of carboplatin in infants treated for localized and unresectable neuroblastoma: Results of the SFOP NBL90 study. Pediatr Blood Cancer, 45, 32-6.
[11] Fischer J, Pohl A, Volland R, et al (2017). Complete surgical resection improves outcome in INRG high-risk patients with localized Neuroblastoma older than 18 months. BMC Cancer, 17,520Kubota M, Yagi M, Kanada S, et al (2004). Long-term follow-up status of patients with Neuroblastoma after undergoing either aggressive surgery or chemotherapy-a single institutional study. J. Pediatr. Surg, 39, 1328-2.

[12] Kubota M, Yagi M, Kanada S, et al (2004). Long-term followup status of patients with Neuroblastoma after undergoing either aggressive surgery or chemotherapy-a single institutional study. J. Pediatr. Surg, 39, 1328-2.

[13] Seeger RC, Reynolds CP, Gallego R, et al (2000). Quantitative tumor cell content of bone marrow and blood as a predictor of outcome in stage IV neuroblastoma: a Children's Cancer Group Study. J Clin Oncol, 18, 4067-6.

[14] Jennifer M., Irene IP, Benjamin AF, et al (2016). Salvage Rates after Progression of High-Risk Neuroblastoma with a Soft Tissue Mass. J Pediatric Surg, 51, 285-8.

[15] Basta ON, Halliday GC, Makin G, et al (2016). Factors associated with recurrence and survival length following a relapse in patients with Neuroblastoma. Br J Cancer, 115, $1048-7$.

[16] Pinto N, Naranjo A, Hibbitts E, et al (2019). Predictors of differential response to induction therapy in high-risk Neuroblastoma: A report from the Children's Oncology Group (COG). Eur J Cancer, 112, 66-9.

[17] Abdel Rahman H, Moussa EA, Zekri WK, et al (2011). Did Salvage ICE Chemotherapy Improve the Outcome in Primary Resistant/Relapsing Stage III/IV Neuroblastoma? J. Egypt. Nat. Cancer Inst, 23, 47-3.

[18] Simon T, Berthold F, Borkhardt A, et al (2011). Treatment and Outcomes of Patients with Relapsed, High-Risk Neuroblastoma? Results of German Trials. Pediatric blood cancer, 56, 578-3.

[19] Illhardt T, Toporski J, Feuchtinger T, et al (2018). Haploidentical Stem Cell Transplantation for Refractory/Relapsed Neuroblastoma. Biol. Blood Marrow Transplant, 24, 1005-2. 